\title{
Penning trap mass measurements of transfermium elements with SHIPTRAP
}

\author{
M. Block • D. Ackermann • K. Blaum • C. Droese • M. Dworschak • M. Eibach • \\ S. Eliseev • T. Fleckenstein · E. Haettner · F. Herfurth • F. P. Heßberger • \\ S. Hofmann · J. Ketelaer • J. Ketter • H.-J. Kluge • G. Marx • M. Mazzocco • \\ Yu. N. Novikov • W. R. Plaß • A. Popeko • S. Rahaman • D. Rodríguez • \\ C. Scheidenberger · L. Schweikhard · P. G. Thirolf • G. K. Vorobyev • C. Weber
}

Published online: 26 January 2010

(C) The Author(s) 2010. This article is published with open access at Springerlink.com

\begin{abstract}
Penning traps are widely used for high-precision mass measurements of radionuclides related to nuclear astrophysics studies and the evolution of nuclear structure far away from stability. With the stopping of secondary beams in gas cells
\end{abstract}

\footnotetext{
M. Block (凶) · D. Ackermann · M. Dworschak · F. Herfurth · F. P. Heßberger · S. Hofmann · H.-J. Kluge · Yu. N. Novikov · C. Scheidenberger · G. K. Vorobyev GSI Helmholtzzentrum für Schwerionenforschung GmbH, 64291 Darmstadt, Germany e-mail:m.block@gsi.de
}

K. Blaum · S. Eliseev $\cdot$ J. Ketter

Max-Planck-Institut für Kernphysik, 69117 Heidelberg, Germany

C. Droese · G. Marx · L. Schweikhard

Ernst-Moritz-Arndt-Universität, 17489 Greifswald, Germany

M. Eibach · J. Ketelaer

Johannes Gutenberg-Universität, 55128 Mainz, Germany

T. Fleckenstein · E. Haettner · W. R. Plaß · C. Scheidenberger Justus-Liebig-Universität, 35390 Gießen, Germany

M. Mazzocco

Dipartimento di Fisica and INFN Sezione di Padova, 35131 Padova, Italy

Yu. N. Novikov

PNPI RAS, Gatchina, Leningrad district 188300, Russia

\section{A. Popeko}

Flerov Laboratory of Nuclear Reactions, JINR, 141980 Dubna, Russia

S. Rahaman

Los Alamos National Laboratory, Los Alamos, NM 87545, USA

D. Rodríguez

Departamento de FAMN, Universidad de Granada, 18071 Granada, Spain

P. G. Thirolf · C. Weber

Ludwig-Maximilians-Universität München, 85748 Garching, Germany 
together with advanced ion-beam manipulation techniques their reach has been extended to rare isotopes of essentially all elements. The Penning trap mass spectrometer SHIPTRAP at GSI Darmstadt has recently demonstrated that even highprecision mass measurements of transfermium elements can be performed despite low production rates of only about one particle per second. This important milestone opens new perspectives for the study of superheavy elements with ion traps.

Keywords Penning trap mass spectrometer • Transactinides • Superheavy elements

\section{Introduction}

The mass is a fundamental property of every atom and contains information about all its constituents and their interactions [1]. Thus, high-precision mass measurements provide a powerful tool for the study of fundamental interactions and are a sensitive probe for the investigation of the nuclear structure evolution far away from stability [2, 3]. In Penning traps single particles can be stored at rest for long times under perturbation-free conditions and high mass resolving powers can be achieved. Therefore, ion traps are ideal devices for mass measurements of highest accuracy and nowadays utilized at radioactive beam facilities worldwide [2, 3].

The application of gas stopping in combination with advanced ion-beam manipulation techniques has extended the reach of Penning traps to reaction products from fusion-evaporation reactions and projectile fragmentation so that rare isotopes of practically all elements are now accessible. Even the elements above fermium can be reached with the Penning trap mass spectrometer SHIPTRAP [4] at GSI. The nuclides in this region of high proton and neutron numbers owe their stability against spontaneous fission to a delicate balance between the repulsive Coulomb force and nuclear attraction. The existence of superheavy elements (SHE) on an island of stability far above the then known nuclides was predicted about forty years ago $[5,6]$. Ever since this prediction has motivated experimental activities trying to reach the island of stability at $N=184$ and $Z=114$ or $Z=120$. Superheavy elements have indeed been synthesized [7] with the latest claims reaching up to $Z=118$ [8]. The recent results suggest that the coast of the island has been reached, but the exact location of its center and its extension are still unknown. The synthesis of new elements above $Z=118$ is very challenging due to extremely low production rates and the need of exotic actinide targets. Moreover, the more neutron-rich nuclides at $N=184$ and beyond cannot be reached at present in complete fusion reactions with stable beams.

Accurate experimental data of nuclear properties such as masses, half-lives and energies of excited states in the region above $Z=100$ are crucial for a sound understanding of the structure of superheavy elements. In particular, binding energies and neutron separation energies that can be derived from masses are important input parameters for theoretical models. However, no direct mass measurements in the region above uranium have been performed until recently. 


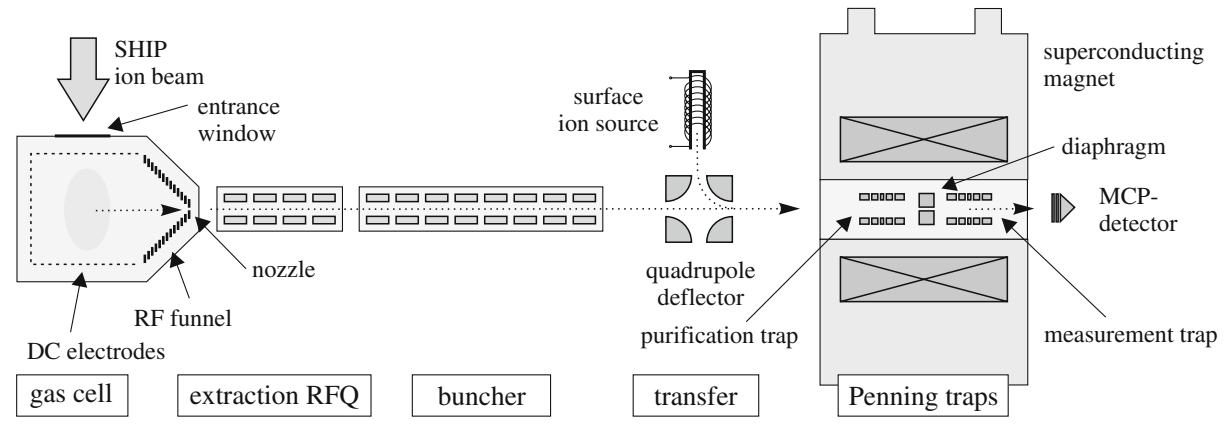

Fig. 1 Schematic SHIPTRAP setup

\section{SHIPTRAP setup}

The Penning trap mass spectrometer SHIPTRAP has been used for mass measurements of more than fifty radionuclides since 2005, contributing to nuclear structure and astrophysics studies [9-11]. A schematic overview of the SHIPTRAP setup is shown in Fig. 1 [4]. The reaction products are kinematically separated from the primary beam in-flight by the velocity filter SHIP [7] and then injected into the SHIPTRAP buffer-gas cell with an energy of a few $10 \mathrm{MeV}$ through a thin titanium window foil. Additional mylar degraders installed about $30 \mathrm{~cm}$ in front of the window are used to tune the stopping range of the nuclides. The reaction products are slowed down and thermalized in high-purity helium at a pressure of 50 mbar and extracted from the gas cell within a few milliseconds by electric fields and gas flow through an extraction nozzle. Depending on their ionization potential the ions remain either singly or doubly charged. The extracted ions are transported by an RF quadrupole (RFQ) ion guide to an RFQ cooler and buncher, where they are cooled, accumulated and bunched. The short bunches are captured in the purification Penning trap inside a 7-T superconducting solenoid where a mass-selective buffer-gas cooling technique is applied to select the nuclides of interest with a mass resolving power of up to 100,000 . The mass-selected ion sample is then transferred through a $1 \mathrm{~mm}$ orifice into the measurement Penning trap that is situated in the same solenoid. A time-of-flight cyclotron-resonance detection technique [12] is used to measure the ions' cyclotron frequency $v_{c}=q B /(2 \pi m)$, from which the mass $m$ of the ion with charge $q$ can be determined. To this end the ions are excited with an RF at a frequency $v$ and their time of flight to an ion detector outside the trap is measured. If $v=v_{c}$ the ions gain maximum kinetic energy resulting in a minimum time of flight to the detector. The frequency $v$ is scanned about the expected cyclotron frequency and a resonance as shown in Fig. 2 is obtained.

\section{Direct mass measurements of transfermium elements}

In the lastest Atomic-Mass Evaluation (AME'03) [13] all masses in the region of elements above fermium were derived from decay links to nuclides with well-known mass. There are, however, some difficulties associated with this approach. While 
Fig. 2 Cyclotron resonance curve of ${ }^{254} \mathrm{No}^{2+}$. The excitation time was $900 \mathrm{~ms}$. The solid line is a fit of the expected line shape to the data points

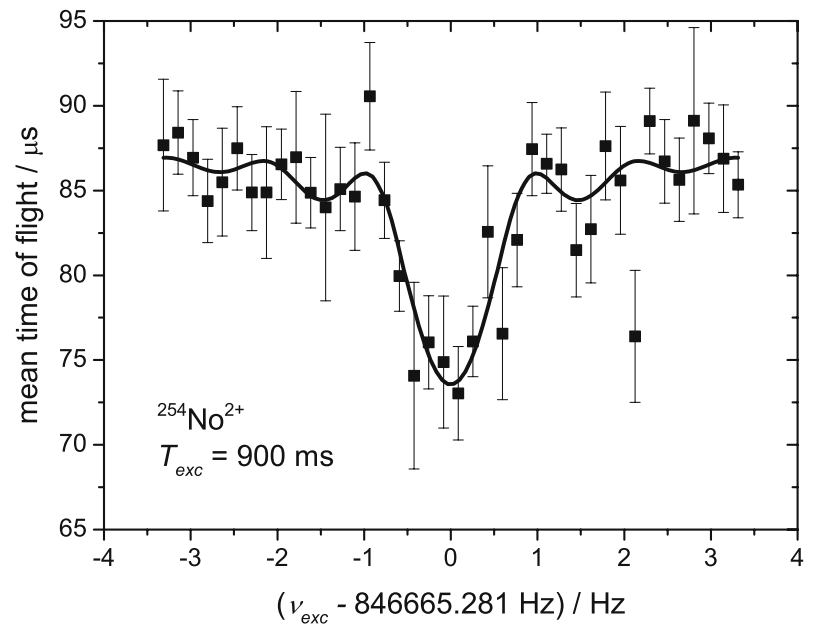

$\alpha$-decay links for even-even nuclides, where the decay occurs between ground states, are reliable, the mass uncertainty for other nuclides accumulates with increasing distance from a so-called primary nuclide, a nuclide whose mass is known from two independent measurements [13]. For odd-even nuclei additional complications arise since the preferred $\alpha$ decay often populates excited states. Then, an accurate mass determination requires the energies of the excited states and their ordering to be well known. In such cases direct mass measurements are a favorable approach to determine the ground state masses unambiguously. Moreover, they can often provide information complementary to decay spectroscopy experiments, in particular for studies of long-lived isomeric states.

Recently, the first direct mass measurements in the region above uranium have been performed with SHIPTRAP. The masses of the three nobelium isotopes ${ }^{252-254}$ No $(Z=102)$ were measured in August 2008 [14]. These nuclides can be produced in the reactions ${ }^{48} \mathrm{Ca}\left({ }^{206-208} \mathrm{~Pb}, 2 \mathrm{n}\right)$ with relatively high cross sections on the order of $1 \mu \mathrm{b}$ corresponding to about one ion per second entering the SHIPTRAP gas cell. Due to the low ionization rate by the incident ion beam and the high cleanliness of the SHIPTRAP gas cell mainly doubly-charged nobelium ions were extracted. Thus, even for the heavy nobelium ions a resolving power of about $1.4 \times 10^{6}$ was obtained in the measurement trap. An example for a resonance of ${ }^{254} \mathrm{No}^{2+}$ is shown in Fig. 2. The measurement times for single nobelium resonances varied between one and four hours depending on the production rate of the nuclide under investigation. ${ }^{254} \mathrm{No}$ is presently the heaviest radionuclide for which a direct mass measurement was performed. The accurate nobelium masses provide reliable reference points in the mass region above $Z=100$. Since their masses are now determined by at least two independent measurements these nobelium nuclides became now primary nuclides in the AME framework. In combination with established $\alpha$ decays the masses of heavier nuclides, many of which are too short-lived for direct mass measurements in a Penning trap, can thus be determined more accurately. The situation is illustrated in Fig. 3 where the decay chains passing through the nobelium isotopes investigated at SHIPTRAP are shown. At present, the weak $\alpha$ decay branch 


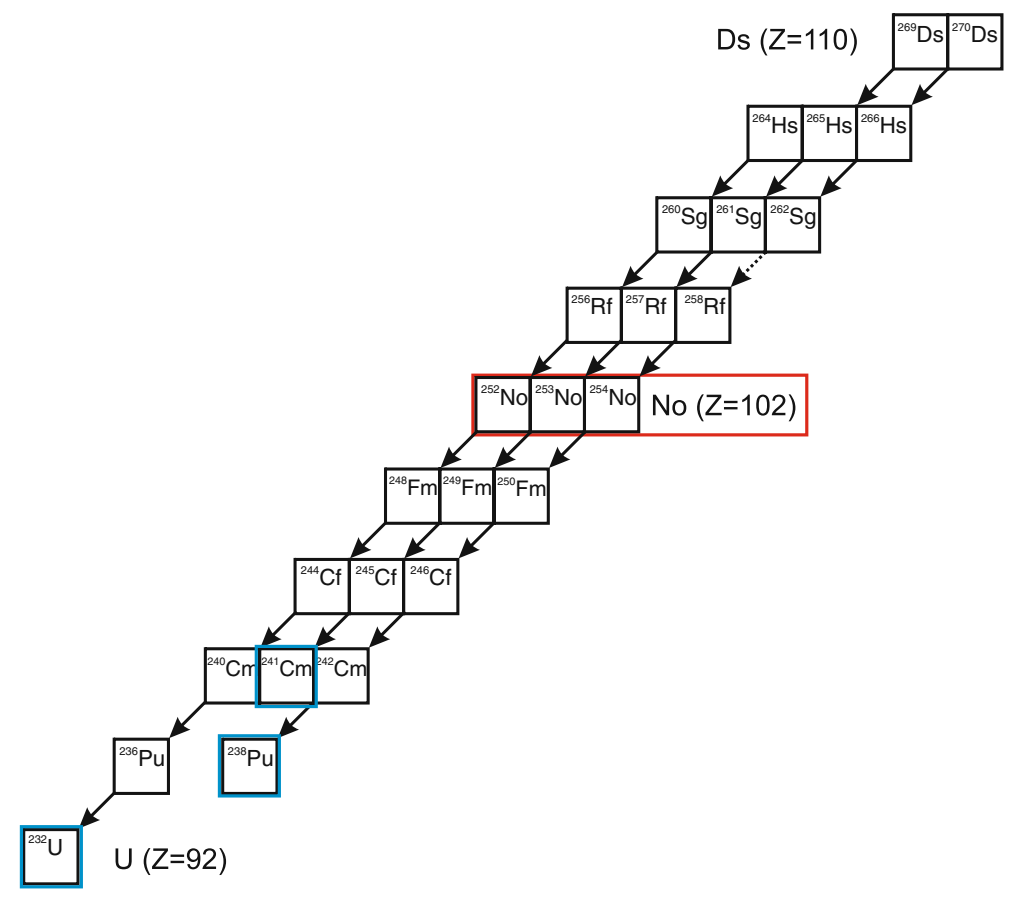

Fig. $3 \alpha$-Decay chains passing through the nobelium isotopes investigated with SHIPTRAP. Primary nuclides of the AME'03 are indicated by a thick frame

in ${ }^{262} \mathrm{Sg}$ (dashed line in Fig. 3) has not been observed, but once this connection will be added, the SHIPTRAP results reach up to ${ }^{270} \mathrm{Ds}(Z=110)$.

\section{Perspectives}

The first direct mass measurements of transfermium elements with SHIPTRAP have demonstrated the feasibility of high-precision experiments with Penning traps even for radionuclides with production rates of only one per second. Future developments such as a cryogenic gas cell [15] will help to push this limit to even lower production rates to extend such experiments to elements with higher $Z$. In addition, the utilization of a non-destructive electronic detection system will allow a mass determination from a single-ion for nuclides with a half-life of about one second or longer. Such a system is presently being developed at TRIGA-TRAP [16]. Figure 4 shows an example of a resonance obtained with the detection circuit equipped with a coil for $A \approx 150$, where a quality factor of $\approx 5,000$ was obtained. These developments will open up interesting perspectives for studies of transactinides with traps. An important issue in superheavy element research is the unambiguous identification of a newly synthesized nuclide. At present it relies on the observation of decay chains that connect a new nuclide to the region of known nuclides. However, this represents a problem for nuclides synthesized in ${ }^{48} \mathrm{Ca}$-induced reactions with actinide targets, where so far the observed decay chains [8] ended in nuclides decaying predominantly 


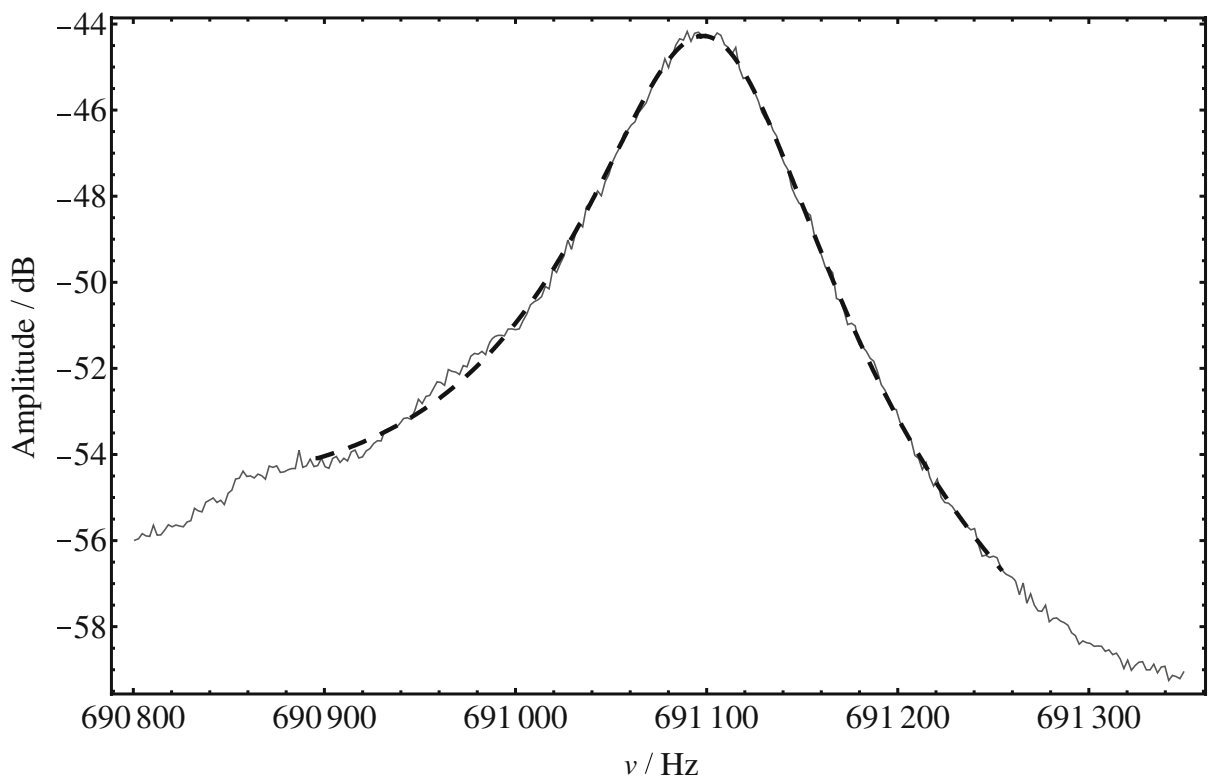

Fig. 4 Resonance curve obtained with the electronic detection system developed for TRIGA-TRAP [16] with a superconducting coil for $A \approx 150$. The dashed line represents a Lorentz profile fitted to the data points. A resonance frequency of $(691,101 \pm 2) \mathrm{Hz}$ was obtained corresponding to a quality factor of $(5.03 \pm 0.03) \times 10^{3}$

by spontaneous fission. In the future laser spectroscopy techniques with their $Z$ selectivity in combination with an $A / q$-selection by mass spectrometry can provide an alternative approach for the identification of superheavy elements, in particular for long-lived nuclides when an identification based on the observation of correlated decay chains will no longer work.

Open Access This article is distributed under the terms of the Creative Commons Attribution Noncommercial License which permits any noncommercial use, distribution, and reproduction in any medium, provided the original author(s) and source are credited.

\section{References}

1. Lunney D., Pearson, J.M., Thibault, C.: Rev. Mod. Phys. 75, 1021 (2003)

2. Blaum, K.: Phys. Rep. 205, 1 (2006)

3. Schweikhard, L., Bollen, G. (eds.): Int. J. Mass Spectrom. 251, (2/3) (2006)

4. Block, M., et al.: Eur. Phys. J., D 45, 39 (2007)

5. Meldner, H.: Ark. Fys. 36, 593 (1967)

6. Mosel, U., Greiner, W.: Z. Phys. 222, 261 (1969)

7. Hofmann, S., Münzenberg, G.: Rev. Mod. Phys. 72, 733 (2000)

8. Oganessian, Yu. Ts., et al.: Phys. Rev., C 69, 054607 (2004)

9. Martín, A., et al.: Eur. Phys. J., A 34, 341 (2007)

10. Weber, C., et al.: Phys. Rev. 78, 054310 (2008) 
11. Rauth, C., et al.: Phys. Rev. Lett. 100, 012501 (2008)

12. König, M., Bollen, G., Kluge, H.-J., Otto, T., Szerypo, J.: Int. J. Mass Spectrom. Ion Process. 142, $95(1995)$

13. Audi, G., Wapstra, A.H., Thibault, C.: Nucl. Phys., A 729, 327 (2003)

14. Block, M., et al.: Nature (in press)

15. Eliseev, S., et al.: Nucl. Instrum. Methods B 266, 4475 (2008)

16. Ketelaer, J. et al.: Eur. Phys. J. A 42, 311 (2009) 